Apidologie, 1979, 10 (4), 313-340.

\title{
ANALYSE POLLINIQUE DES MIELS DU QUÉBEC
}

\section{Pollenanalyse von Honigen aus Quebec}

\author{
Marie-José FELLER-DEMALSY et Yvan LAMONTAGNE* \\ avec la collaboration technique \\ de Denise MARQUIS et de Johanne PARENT \\ Université du Québec à Rimouski, 300, av. des Ursulines \\ Rimouski (Québec) G5L 3Al; Canada
}

SUMMARY

POLLEN ANALYSIS OF HONEYS FROM QUÉBEC

Microscopic pollen analysis was made from 206 honey samples collected in all the beekeeping areas of Québec during 1974 and 1975. Four forms of pollens were shown to be of major importance in the composition of the honeys : Trifolium hybridum, Solidago spp., fruit trees and Salix spp. Geographic indicators were found. The main ones are : Lythrum salicaria (primarily on the north bank of the SaintLawrence river), Sanguisorba canadensis and Cornus stolonifera (east of Québec), Lotus corniculatus (west of the province), Fagopyrum sagittatum (Montréal and Mauricie region), Melilotus spp. (regions not bordering Saint-Lawrence river). A table was made from the subdivision of Quebec into six large zones in which the honeys apparently have their proper characteristics (Table 7).

\section{RESUME}

L'étude microscopique des pollens a été effectuée sur 206 échantillons de miel des années 1974 et 1975 provenant de toutes les régions du Québec où se pratique lapiculture. Quatre formes de pollens se sont montrées d'importance majeure dans les miels : Trifolium hybridum, Solidago spp., les arbres fruitiers et Salix zpp. Des indicateurs géographiques ont pu être décelés. Les principaux sont : Lythrum salicaria (surtout la rive Nord du Saint-Laurent), Sanguisorba canadensis et Cornus stolonifera (Québec Oriental). Lotus corniculatus (l'Ouest de la province), Fagopyrum sagitfatum (région de Montréal et Mauricie), Melilotus spp. (régions écartées du Saint-Laurent). La subdivision du Québec en six grandes zones dont les miels paraissent posséder des caractéristiques propres a été esquissée (tableau 7).

* Laboratoire d'expertise et d'analyse alimentaire, Ministère de l'Agriculture du Québec. Complexe scientifique, 2700, bd Einstein, Sainte-Foy, Québec, G1P 3W8. Canada. 


\section{INTRODUCTION}

Bien que l'apiculture soit en pleine expansion au Québec, aucune étude systématique de la composition pollinique des miels n'a encore été entreprise à notre connaissance. Louveaux (1966) a effectué l'analyse pollinique d'un miel du Québec (probablement du nord de Montréal) parmi un lot de miels canadiens. Mais les renseignements dont nous disposons se rapportent plutôt à la qualité mellifère de la flore qu'à la composition des miels. Ainsi, Снавот (1948) a établi une liste de plantes mellifères du Québec dont il décrit les caractéristiques mellifères. Pellet (1977) signale les principales sources botaniques des miels pour le Canada et les États-Unis mais il semblerait que ces données ne soient qu'exceptionnellement étayées par l'étude pollinique de miels. Enfin des renseignements épars sur les caractéristiques mellifères de la végétation québécoise sont fournis dans la flore de frère MARIE-VICTORIN (1964).

L'étude des miels du Québec est particulièrement intéressante de par la situation géographique de la province. Situé au Nord-Est du Continent et traversé par un grand fleuve, le Québec offre sur son territoire une certaine variété climatique. Toutefois, l'apiculture n'est pratiquée que dans la partie méridionale, la localisation des ruchers ne dépassant pas $49^{\circ}$ de latitude Nord sauf quelque peu sur la côte Nord de la Gaspésie. Par ailleurs, l'agriculture qui est fortement développée dans l'extrême sud du territoire et surtout le long des rives du Saint-Laurent l'est moins ailleurs oủ prédominent des étendues recouvertes de végétation spontanée et de forêts. 11 est à noter que l'apiculture n'est pratiquée que dans les régions habitées et que celles-ci n'occupent qu'une petite partie de la province.

Le présent travail est consacré principalement à l'étude de l'origine géographique et botanique des miels du Québec et à la recherche des principales caractéristiques qui permettent d'identifier les miels récoltés dans les différentes régions du territoire.

\section{MATÉRIEL ET MÉTHODES}

L'étude a porté sur 206 échantillons de miel dont une moitié environ à été récoltée en 1974 et l'autre en 1975 et prélevés par les services d'inspection du Ministère de l'Agriculture pour la plupart dans les réservoirs ou les apiculteurs entreposent le miel après son extraction des rayons. Il est à noter que, au Québec, le mode de récolte du miel par l'apiculteur (extraction et filtration) n'est pas réglementé, pas plus que dans le reste du Canada. Mais au Québec, à l'encontre d'autres provinces surtout de l'Ouest, l'apiculture est artisanale et le plus grand soin est apporté à la qualité du produit, c'est-à-dire à la préservation de ses qualités naturelles. Les apiculteurs n'effectuent pas de mélange de miels d'origines diverses et prennent soin de séparer les miels qui présentent des différences de couleur.

Ces échantillons proviennent de 51 comtés sur les 74 où se pratique l'apiculture mais appartiennent à toutes les régions du Québec au nombre de 12. La subdivision en comtés a été effectuée d'après le Répertoire Géographique du Québec (1969). Le tableau 1 donne la répartition de l'échantillonnage, le nombre d'échantillons étudiés par comté et par région, le pourcentage d'apiculteurs dont proviennent les échantillons évalué par région (établi d'après les données du Bureau de la Statistique du Québec, 1974) et l'importance que représente l'échantillonnage de chaque région par rapport à l'ensemble. La figure 1 en montre la 
TABL. 1. - Répartition de l'échantillonnage.

Les numéros entre parenthèses permettent la localisation des comtés sur la figure 1.

TAB. 1. - Verteilung der Proben.

Die Zahlen in Klammer ermöglichen die Auffindung der Bezirke in Abb. 1.

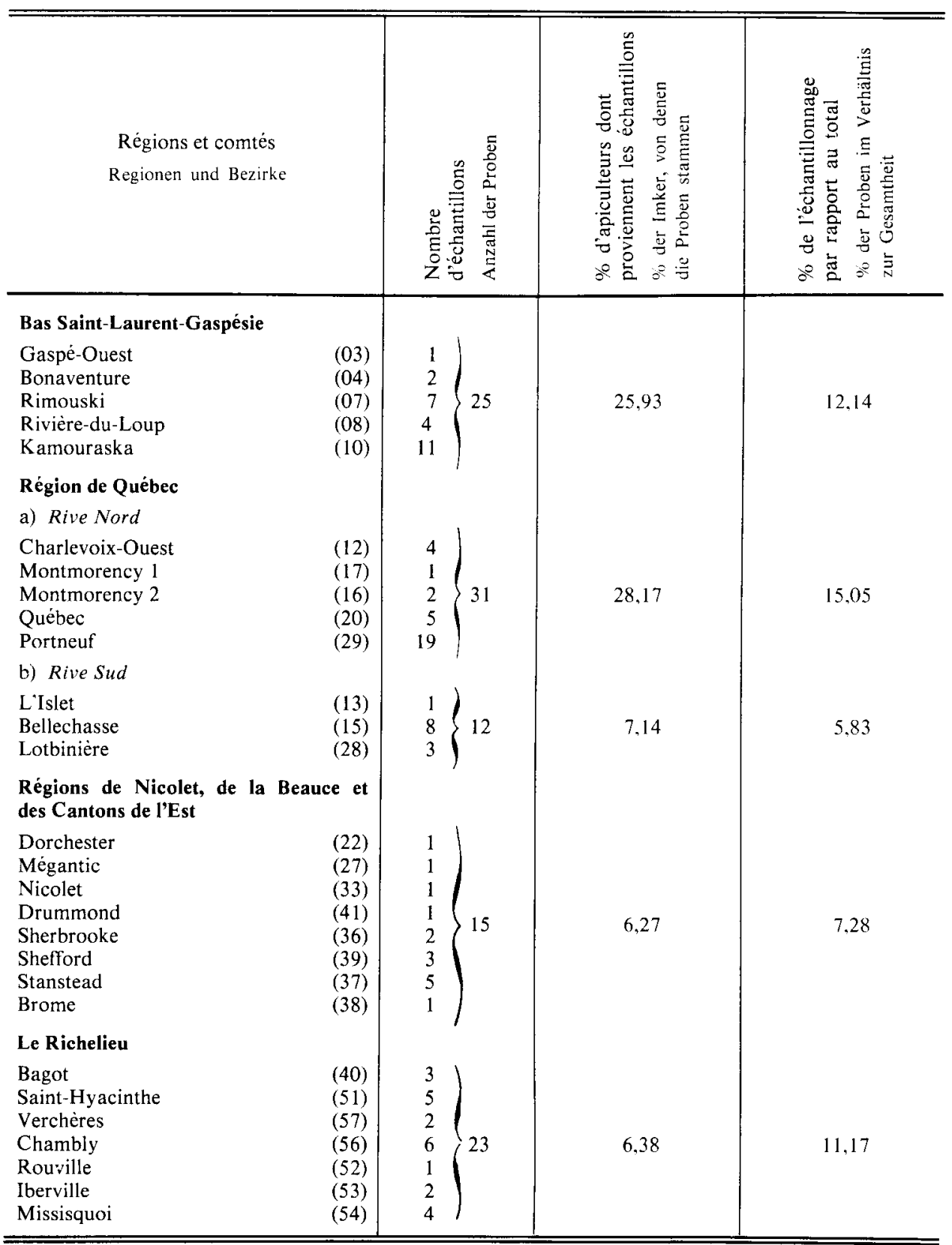




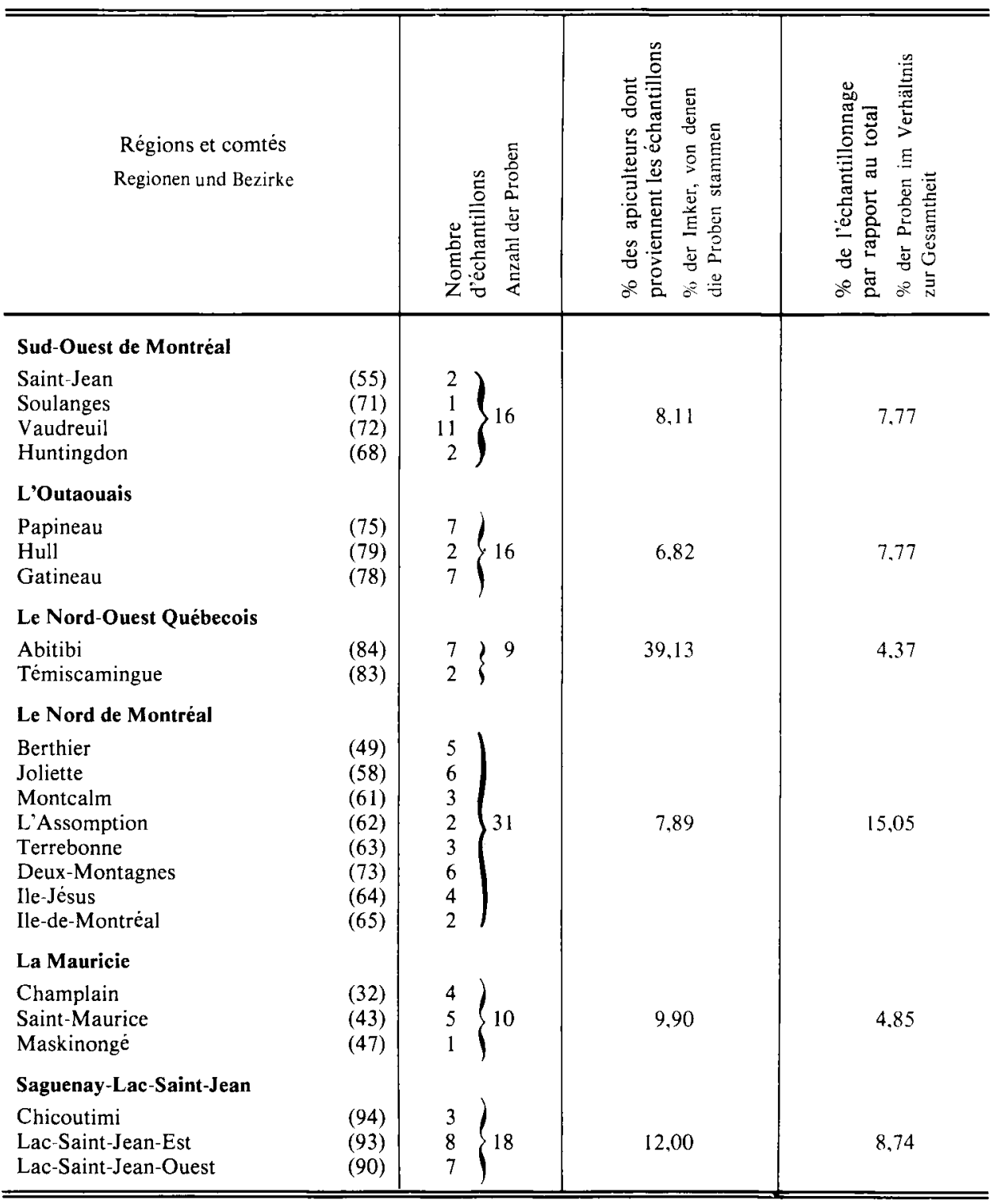

répartition géographique. Pour les besoins du travail, la région de Québec, assez étendue, a été divisèe en deux parties, la rive Nord et la rive Sud qui nous paraissent assez différentes au point de vue de l'environnement et des caractéristiques géologiques et géographiques. Les régions de Nicolet et de la Beauce ont été jointes à celles des Cantons de l'Est vu le petit nombre d'échantillons dont nous disposions et l'uniformité apparente des conditions de milieu. Des échantillons de 1974 et de 1975 sont représentés dans chacune des 11 régions ainsi définies, sauf dans le Nord-Ouest Québécois d'où proviennent uniquement des miels de 1975.

Les échantillons ont été traités selon la méthode de la Commission Internationale de Botanique apicole décrite par LouveauX, MAURIZIO et VoRwOHL (1970). La coloration des pollens a été effectuée à la 
FIG. 1. - Répartition géographique des échantillons étudiés.

Chaque numéro correspond à un comté (voir tableau 1) dont des miels ont fait l'objet de cette étude.

Авв. 1. - Geographische Verteilung der untersuchten Proben.

Jede Zahl entspricht einem Kreis (s. Tab. 1), dessen Honige in dieser Arbeit untersucht wurden.

$\mathrm{O}$ : Herkunft der Probe

- - : Grenze der Region

. . . . : Grenze des Bezirks

-... : Staatsgrenze

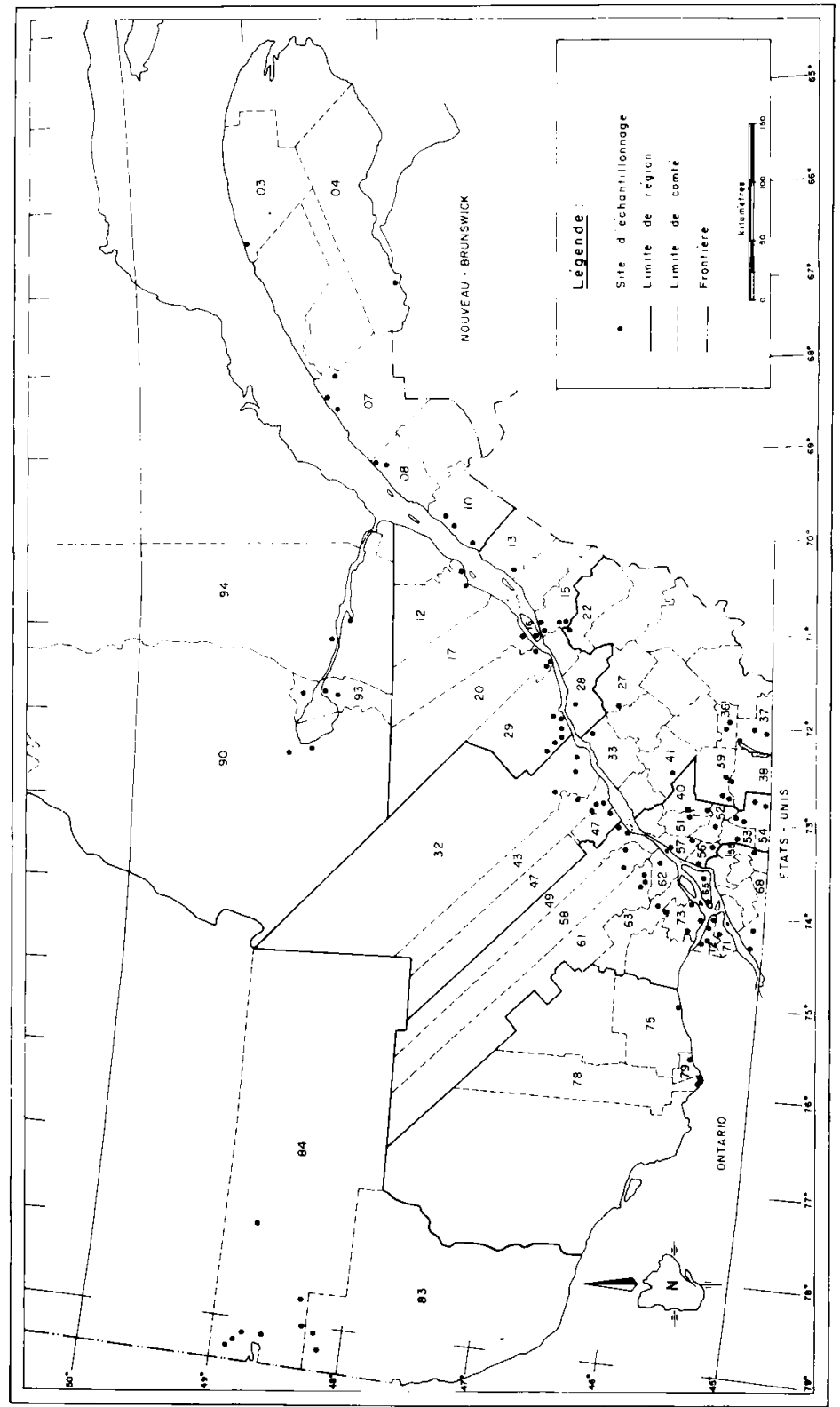


fuchsine basique incluse dans le milieu de montage, la glycérine-gélatine $(0.5 \mathrm{ml}$ de solution alcoolique de fuchsine basique à $0,1 \%$ dans $10 \mathrm{ml}$ de glycérine-gélatine). Les préparations ont finalement étć lutées à la paraffine. Les analyses ont été effectuées sur des quantités de 10 ou $15 \mathrm{~g}$ de miel d'après la richesse en pollen du miel. Lors du montage, une bonne homogénéisation du matériel sur la lame a permis d'obtenir une bonne répartition des grains; des accumulations de pollens n’ont ainsi jamais été observées. ce qui améliore la qualité des observations et la valeur des résultats. Les observations microscopiques ont été effectuées à $400 \mathrm{x}$. Les spectres ont été établis à partir de 100 grains de pollen. Les pourcentages ont été évalués par groupes : les pollens dominants (plus de $45 \%$ ), les pollens d'accompagnement (16-45\%), les pollens isolés importants $(3-15 \%)$ et les pollens isolés rares $(<3 \%)$. Les indicateurs de miellat autres que certains pollens n'ont pas été pris en considération dans ce travail.

\section{RÉSULTATS}

\section{Le nombre de grains de pollen dans les miels du Québec}

Le comptage du nombre de grains par $10 \mathrm{~g}$ de miel a montré que les miels pauvres en pollen dominent au Québec bien que suivis de près par les miels à quantité moyenne de grains : $55 \%$ des miels possèdent moins de 20000 grains, $41 \%$ ont un nombre moyen de grains et seulement $4 \%$ dépassent les 100000 grains. Dans chaque région considérée individuellement (tableau 2), ce sont encore les miels pauvres qui dominent

TABL. 2. - Nombre de grains de pollen dans les micls du Qućbec et de chaque région: pourcentage de chaque catégorie.

TAв. 2. - Anzahl der Pollenkörner in den Honigen lon Quebec und von jeder Region: Prozentsatz jeder Kategorie.

\begin{tabular}{|c|c|c|c|}
\hline $\begin{array}{l}\text { Régions } \\
\text { Regionen }\end{array}$ & $\begin{array}{c}\text { Pauvre } \\
(<20000) \\
\text { Gering }\end{array}$ & $\begin{array}{c}\text { Moyen } \\
(20000-100000) \\
\text { Mittel }\end{array}$ & $\begin{aligned} & \text { Riche } \\
&(>100000) \\
& \text { Reich }\end{aligned}$ \\
\hline Bas Saint-Laurent-Gaspésie & 80 & 16 & 4 \\
\hline $\begin{array}{l}\text { Région de Québec } \\
- \text { Rive Nord } \\
\text { Nordufer } \\
- \text { Rive sud } \\
\text { Südufer }\end{array}$ & $\begin{array}{l}67,74 \\
66,67\end{array}$ & $\begin{array}{l}32,26 \\
33,33\end{array}$ & - \\
\hline Régions Nicolet, Beauce et Cantons de l'Est & 53,33 & 40 & 6,67 \\
\hline Le Richelieu & 60,87 & 34,78 & 4,35 \\
\hline Sud-Ouest de Montréal & 18,75 & 75 & 6.25 \\
\hline Outaouais & 37,50 & 62,50 & - \\
\hline Nord-Ouest Québecois & 66,67 & 22,22 & 11,11 \\
\hline Nord de Montréal & 51,61 & 41,94 & 6,45 \\
\hline La Mauricie & 70 & 30 & - \\
\hline Saguenay-Lac-Saint-Jean & 22.22 & 66,67 & 11.11 \\
\hline $\begin{array}{l}\text { Moyenne pour le Québec } \\
\text { Mittelwert für Quebec }\end{array}$ & 54,85 & 40.78 & 4,37 \\
\hline
\end{tabular}


sauf au Sud-Ouest de Montréal, dans l'Outaouais et au Saguenay-Lac-Saint-Jean où les miels à nombre moyen de grains sont les plus nombreux. C'est dans cette dernière région que se rencontrent les miels les plus riches (comté Lac-Saint-Jean-Est de 250000 à 350000 grains). Les miels les plus pauvres (moins de 1000 grains) se retrouvent dans un comté du Bas Saint-Laurent (un échantillon dans le comté de Kamouraska) et dans la région de Nord de Montréal (Comtés de Berthier, de l'Asseomption et de l'Ile-Jésus; dans deux échantillons provenant de ces deux derniers comtés, les spectres n'ont pu être établis). Il est à noter que ces chiffres sont à considérer avec beaucoup de prudence, les procédés de récolte des apiculteurs étant très variables au Québec puisqu'il n'y existe aucune réglementation, tant provinciale que fédérale à ce sujet.

\section{Le nombre de formes de pollens dans les miels du Québec}

Dans l'ensemble des miels analysés, 56 formes de pollen ont pu être identifiées. Ce nombre correspond aux formes identifiables mais est en fait inférieur à celui de formes réellement présentes dans ces miels. Dans plusieurs cas, nous avons dû nous limiter à la forme du genre, l'identification des espèces étant hasardeuse au grossissement utilisé. La distinction des deux genres Aster spp. et Solidago spp. n'a pas été faite car leurs formes de pollen diffèrent peu. Ceux-ci seront rapportés sous le nom Solidago spp., la quantité de formes d'Aster spp. étant apparemment infime dans les miels. Diverses Rosacées arbustives ont été regroupées sous la dénomination "arbres fruitiers " qui pour plusieurs auteurs incluent seulement les formes de pollen de Pyrus spp. et Prunus spp. Nous y avons joint aussi Crataegus spp. et Amelanchier spp. dont les formes ne sont pas toujours bien distinctes des genres précédents. Les pollens de Trifolium hybridum et de Trifolium repens très semblables ont été classés sous le nom de Trifolium hybridum apparemment plus abondant dans la flore du territoire. Cette catégorie de formes pourrait aussi inclure une faible quantité de Medicago spp. dont la structure de l'exine se distingue mal au grossissement où s'effectuent les comptages et qui, bien qu'assez abondant dans la flore, n'a jamais pu être observé avec certitude dans les miels. Les formes de pollen qui ne permettent pas une identification certaine et les inconnues qui présentent les caractères de la famille ont été regroupées sous la rubrique "autres formes" dans chaque famille. Enfin quelques formes inconnues qui ne peuvent être rattachées à aucune famille ont été mises en queue de liste.

Le tableau 3 donne le spectre pollinique des 204 échantillons analysés, ordonnés par comtés et regroupés par régions.

L'examen des catégories de nombres de formes montre que, au Québec, $64 \%$ des miels appartiennent à la catégorie des 6-10 formes, $30 \%$ à la catégorie des 10 15 formes et seulement $4 \%$ à la catégorie $1-5$ formes et $1,5 \%$ à la catégorie 16 20 formes. Dans le Bas Saint-Laurent-Gaspésie et dans la région de Québec rive Nord se retrouvent à peu près les mêmes proportions (tableau 4). Dans la Mauricie domine la catégorie 11-15 formes (50\%). La catégorie 16-20 formes est limitée au Bas SaintLaurent-Gaspésie (maximum 18), dans la région de Québec rive Nord (maximum 17) et 
en Mauricie (maximum 16). La catégorie 1-5 formes se retrouve dans le Bas SaintLaurent-Gaspésie, dans la région de Québec rive Nord, dans le Richelieu, au Nord de Montréal et au Saguenay-Lac-Saint-Jean.

La moyenne de nombre de formes de pollen pour le Québec est de 9,43 par échantillon de miel (Tableau 4). Des nombres moyens inférieurs à cette moyenne ont été trouvés dans les miels des régions situées au Nord du territoire étudié (le Nord-Ouest Québécois et le Saguenay-Lac-Saint-Jean) et ceux des régions les plus méridionales (de la région du Sud-Ouest de Montréal, du Richelieu, de Nicolet-Beauce-Cantons de l'Est et de Québec rive Sud). Les autres régions ont fourni des miels dont la moyenne de formes dépasse la moyenne de l'ensemble du territoire. La figure 2, qui donne les nombres moyens de formes de pollen par miel évalués par comtés, montre que les nombres les plus élevés se retrouvent dans les régions riveraines du Saint-Laurent et de la rivière Ottawa.

\section{Le spectre de fréquence des formes de pollen dans les miels du Québec}

Les formes de pollen présentes dans les miels étudiés peuvent être subdivisées en quatre groupes d'après la fréquence de leur répartition dans les miels (figure 3). Le premier groupe comprend des formes très fréquentes présentes dans $50 \%$ des miels, il s'agit de : Trifolium hybridum (trèfle alsike) (95\%), Solidago spp. (verge d'or) (83\%), Vicia cracca (vesce jargeau) $(70 \%)$, arbres fruitiers ( $71 \%$ ) et Trifolium pratense (trèfle rouge) $(56 \%)$. Toutes sont des plantes nectarifères, pollinifères et entomophiles. Les formes fréquentes présentes dans $20-50 \%$ des miels sont Salix spp. (saule) (49,5\%), les Graminées (44,6\%), Brassica spp. (chou et moutarde) (43\%), Melilotus spp. (Mélilot), Lotus corniculatus (lotier corniculé) $(29,5)$, Fagopyrum sagittatum (sarrazin) $(27,5 \%)$, Rubus spp. (framboisier) (24\%), Hieracium spp. (épervière) $(22,5 \%)$ et Lythrum salicaria (salicaire) (21,5\%). La plupart sont des plantes nectarifères, pollinifères et entomophiles sauf les Graminées qui sont anémophiles mais dont le pollen et le miellat sont récoltés par les abeilles (PeLlet, 1977). Le groupe des formes peu fréquentes présentes dans $10-20 \%$ des miels comprend des Crucifères, Sanguisorba canadensis (sanguisorbe

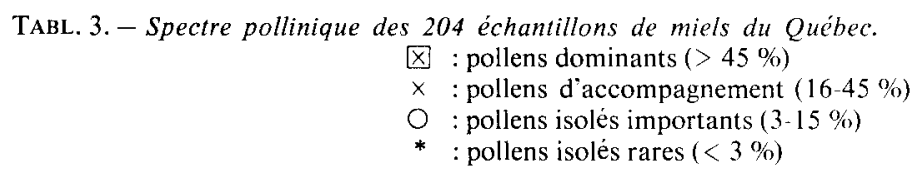

TAB. 3. - Pollenspektrum von 204 Honigproben von Quebec.

凶] : Leitpollen $(>45 \%)$

$x$ : Begleitpollen $(16-45 \%)$

0 : Häufiger Einzelpollen (3-15\%)

* : Seltener Einzelpollen $(<3 \%)$

autres formes : andere Formen

arbres fruitiers : Obstbäume

formes diverses : verschiedene Formen

formes inconnues : unbekannte Formen 


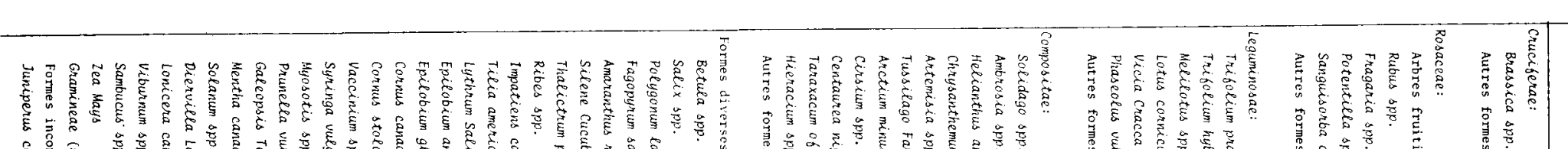

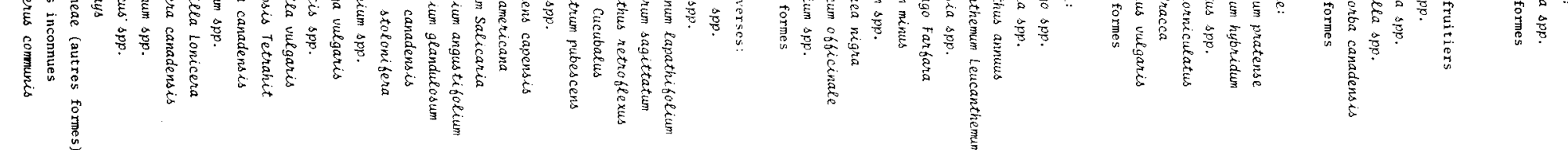

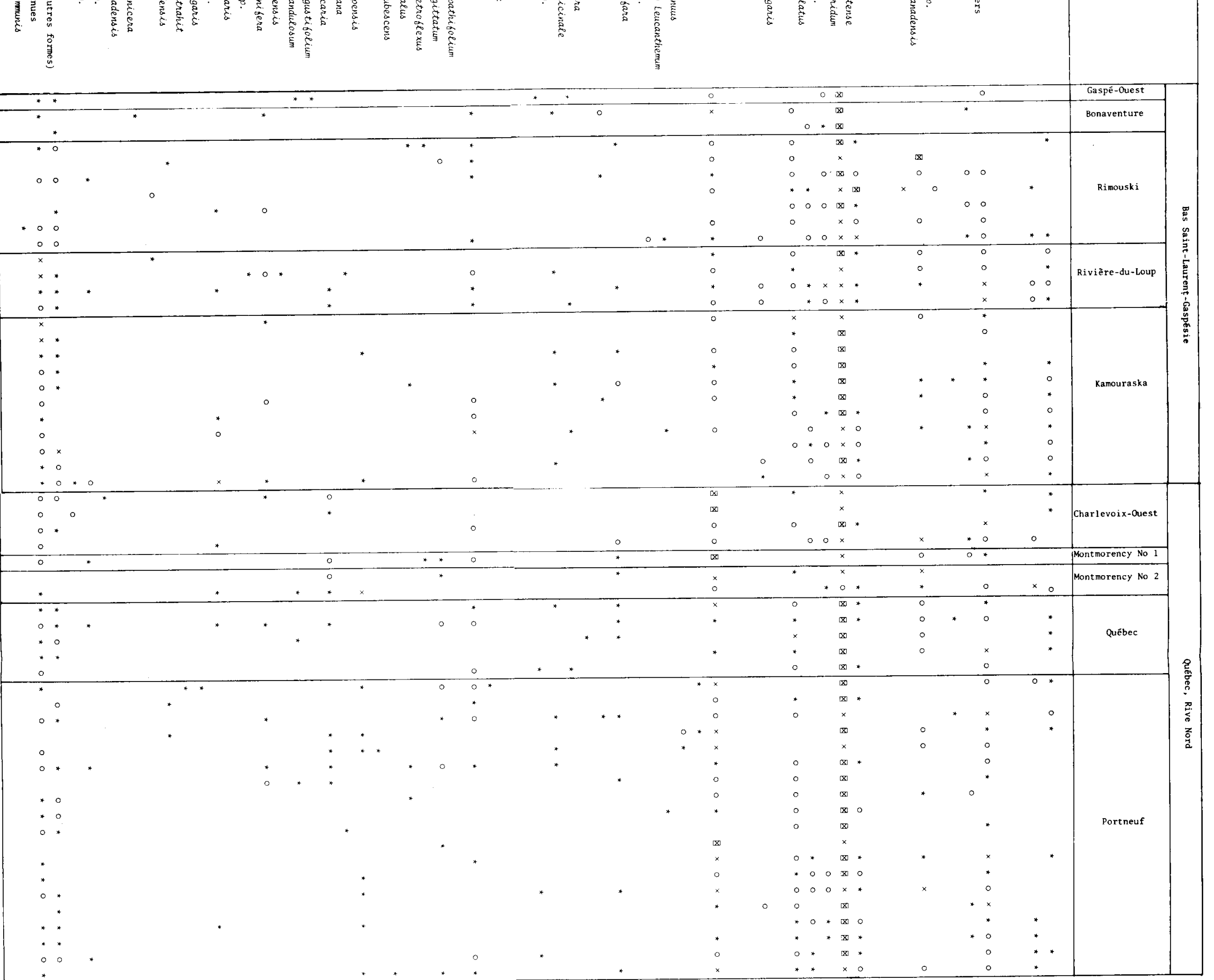

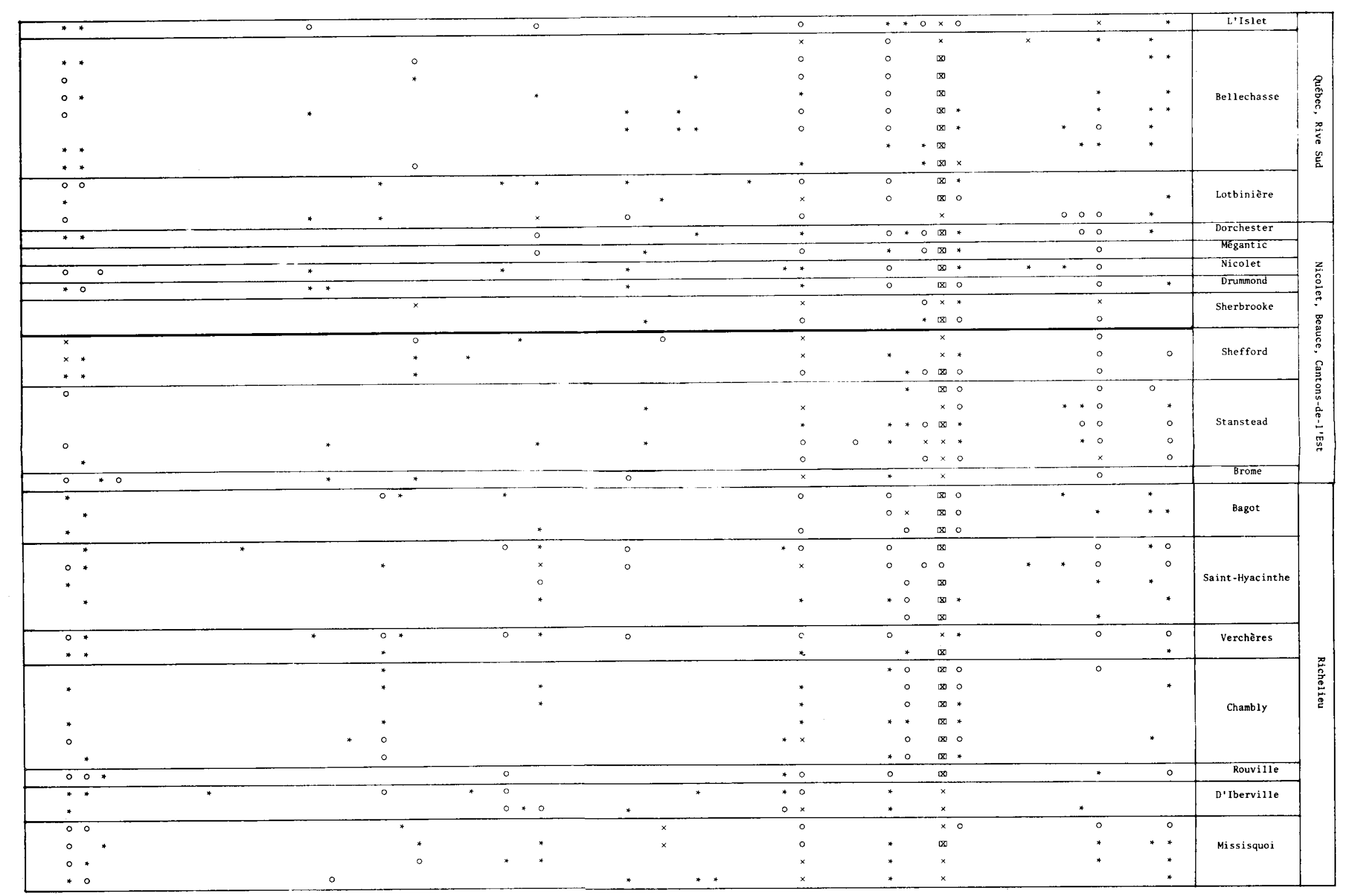



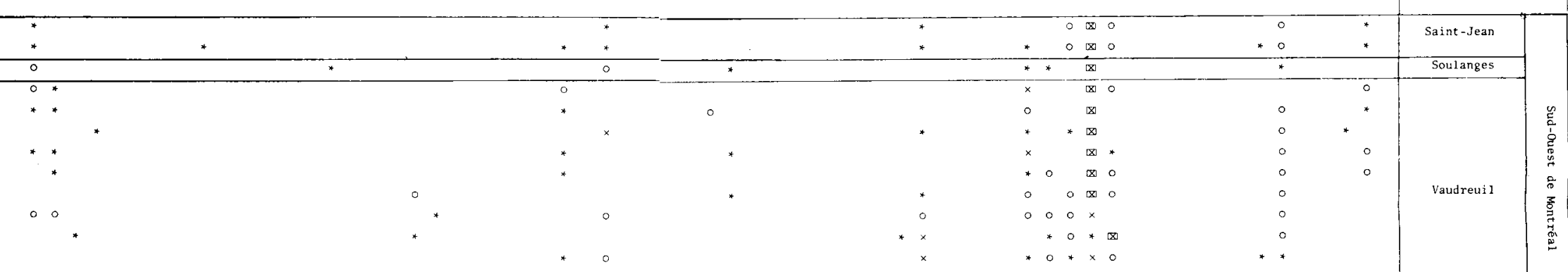




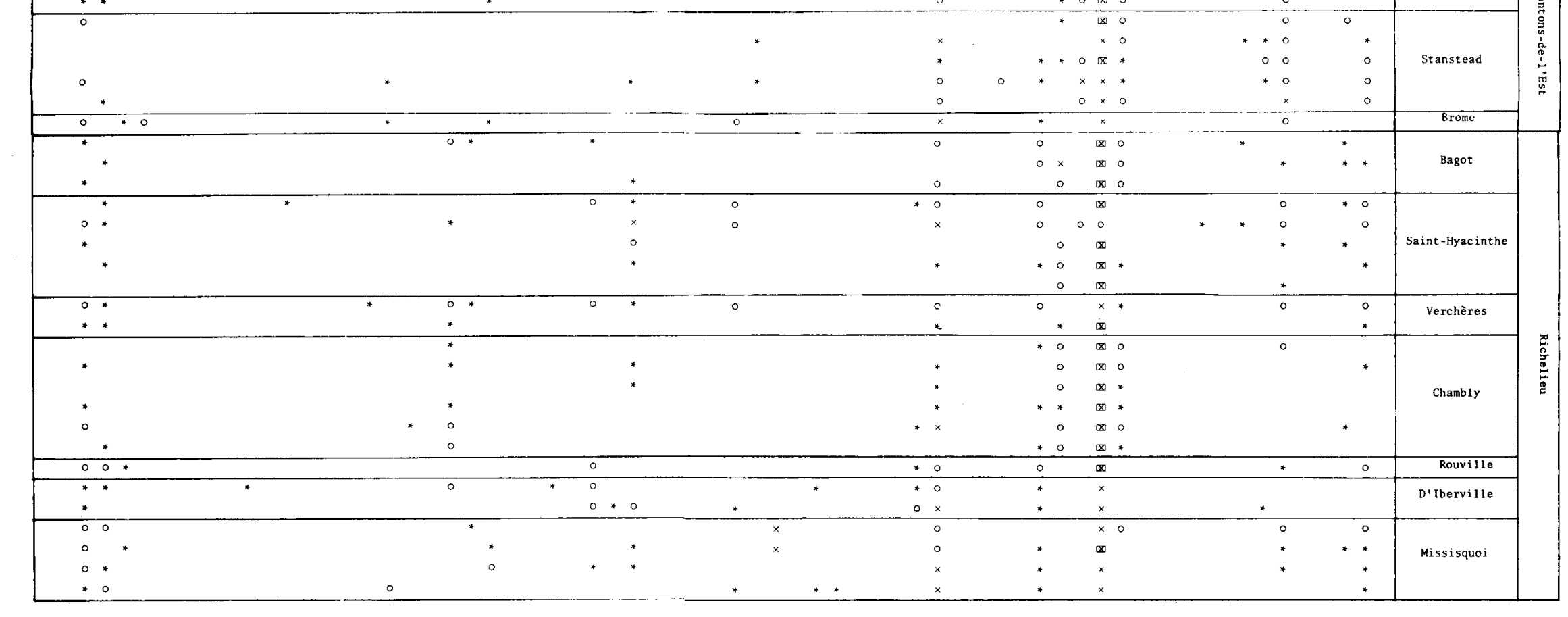

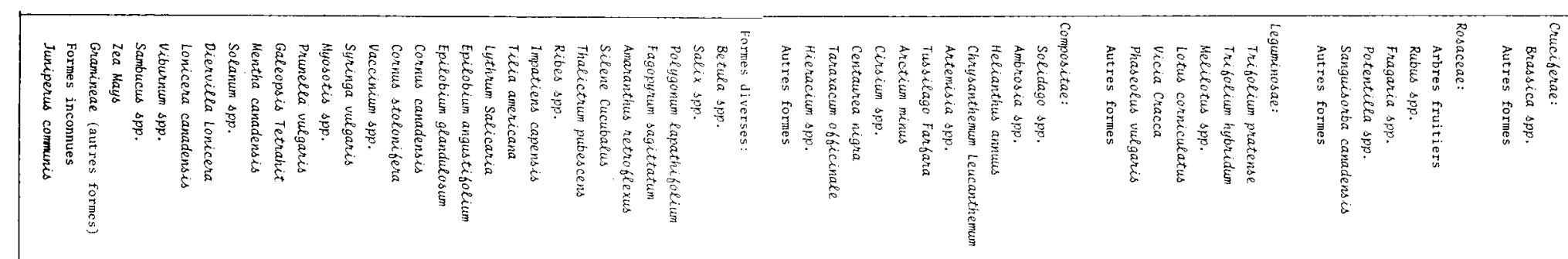

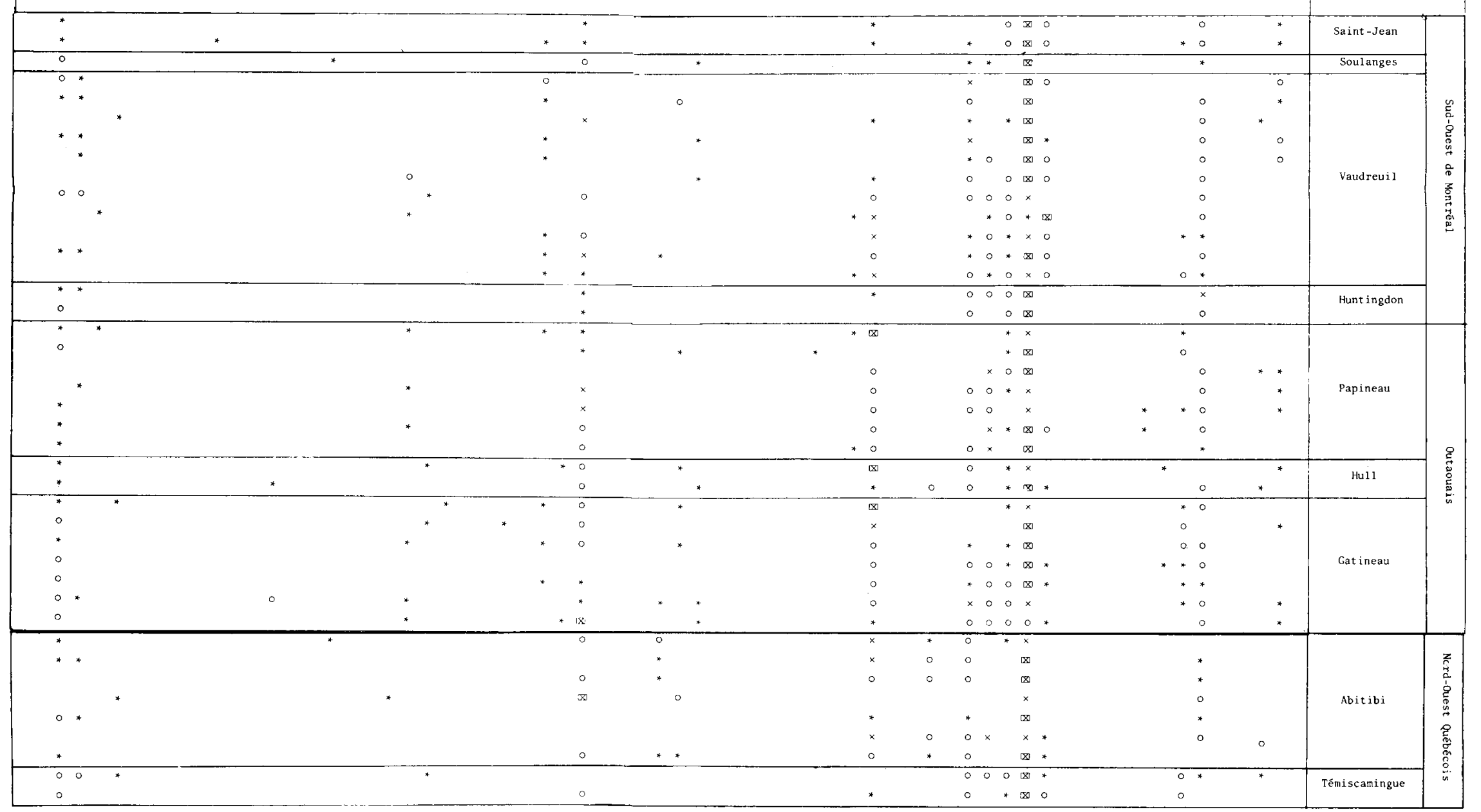

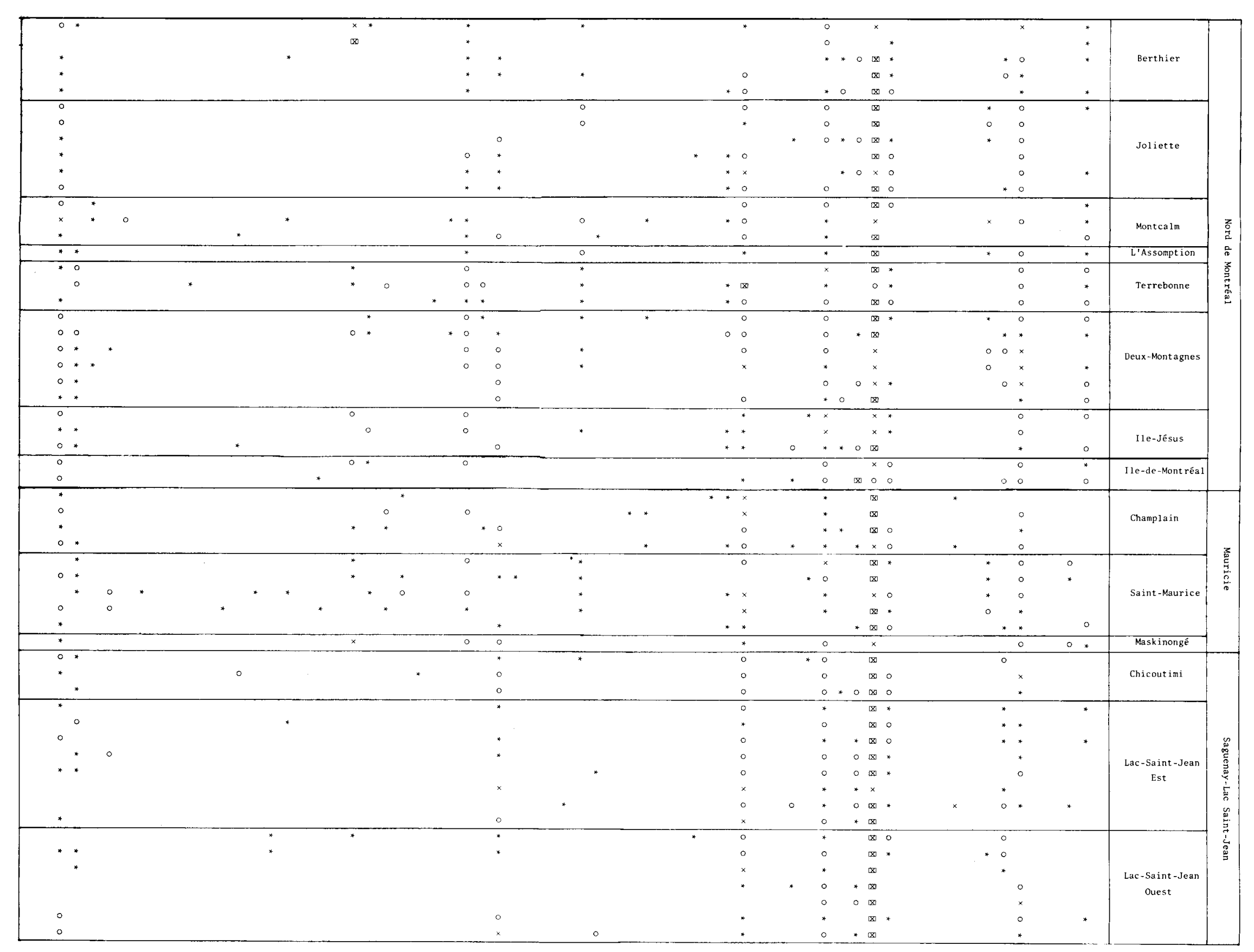


TABL. 4. - Pourcentage des différentes catégories de nombre de formes de pollen par miel et nombre moven

de formes de pollen par miel évalués par région et pour le Québec.

TAв. 4. - Prozentsatz der verschiedenen Kategorien der Anzahl

der Pollenformen pro Honig und mittlere Anzahl der Pollenformen pro Honig, berechnet pro Region und für Quebec.

\begin{tabular}{|c|c|c|c|c|c|c|}
\hline \multirow[t]{2}{*}{$\begin{array}{l}\text { Régions } \\
\text { Regionen }\end{array}$} & \multirow{2}{*}{ 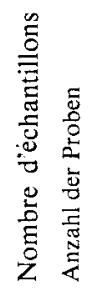 } & \multicolumn{4}{|c|}{$\begin{array}{l}\text { Catégories de nombre de formes } \\
\text { de pollen dans les miels } \\
\text { Kategorien der Anzahl der Pollenformen } \\
\text { in den Honigen }\end{array}$} & \multirow{2}{*}{$\begin{array}{l}\text { Nombre moyen de } \\
\text { formes de pollen } \\
\text { par miel } \\
\text { Mittlere Anzahl } \\
\text { der Pollenformen } \\
\text { pro Honig }\end{array}$} \\
\hline & & $1-5$ & $6-10$ & $11-15$ & $16-20$ & \\
\hline & & $\%$ & $\%$ & $\%$ & $\%$ & \\
\hline Bas Saint-Laurent-Gaspésie & 25 & 8 & 56 & 32 & 4 & 10.28 \\
\hline $\begin{array}{l}\text { Région de Québec } \\
- \text { Rive Nord } \\
\text { Nordufer }\end{array}$ & 31 & 3,23 & 58,06 & 35,48 & 3,23 & 9,90 \\
\hline $\begin{array}{l}\text { - Rive Sud } \\
\text { Südufer }\end{array}$ & 12 & - & 66.67 & 33.33 & - & 8.75 \\
\hline $\begin{array}{l}\text { Régions de Nicolet, Beauce et } \\
\text { des Cantons de l'Est }\end{array}$ & 15 & - & 73,33 & 26,66 & - & 9,06 \\
\hline Le Richelieu & 23 & 8,70 & 69,57 & 21,74 & - & 8,65 \\
\hline Sud-Ouest de Montréal & 16 & - & 81,25 & 18,75 & - & 8.93 \\
\hline Outaouais & 16 & - & 56,25 & 43,75 & - & 10,25 \\
\hline Nord-Ouest Québecois & 9 & - & 88,89 & 11,11 & - & 8.11 \\
\hline Nord de Montréal & 29 & 3,45 & 51,72 & 44.83 & - & 10.48 \\
\hline La Mauricie & 10 & - & 40 & 50 & 10 & 11,40 \\
\hline Saguenay-Lac-Saint-Jean & 18 & 11,11 & 83,33 & 5,56 & - & 8 \\
\hline $\begin{array}{l}\text { Moyenne pour le Québec } \\
\text { Mittelwert für Quebec }\end{array}$ & & 4 & 64 & 30.5 & 1.5 & 9.43 \\
\hline
\end{tabular}

du Canada), A mbrosia spp. (ambroisie ou herbe à poux), Impatiens capensis (impatiente du cap ou chou sauvage), Fragaria spp. (fraisier), Cornus stolonifera (cornouiller stolonifère ou hart rouge) et Arctium minus (bardane mineure ou tabac du diable). Ce sont des plantes nectarifères entomophiles et pollinifères sauf les deux premières, seulement pollinifères. Enfin un dernier groupe comprend 35 formes rares dont 12 sont nectarifères et pollinifères, 9 sont nectarifères, 2 pollinifères et 8 non nectarifères ou mal connues. Dans l'ensemble, la majorité des formes présentes appartiennent à des plantes nectarifères et (ou) pollinifères et entomophiles. Une restriction pourrait être faite pour Trifolium pratense dont la présence dans les miels d'après PELLET (1977) ne serait pas nécessairement représentative de nectar mais de miellat.

La répartition des formes de pollen dans les miels diffère quelque peu d'après les régions comme en témoigne le tableau 5 qui donne l'indice de fréquence des formes de 

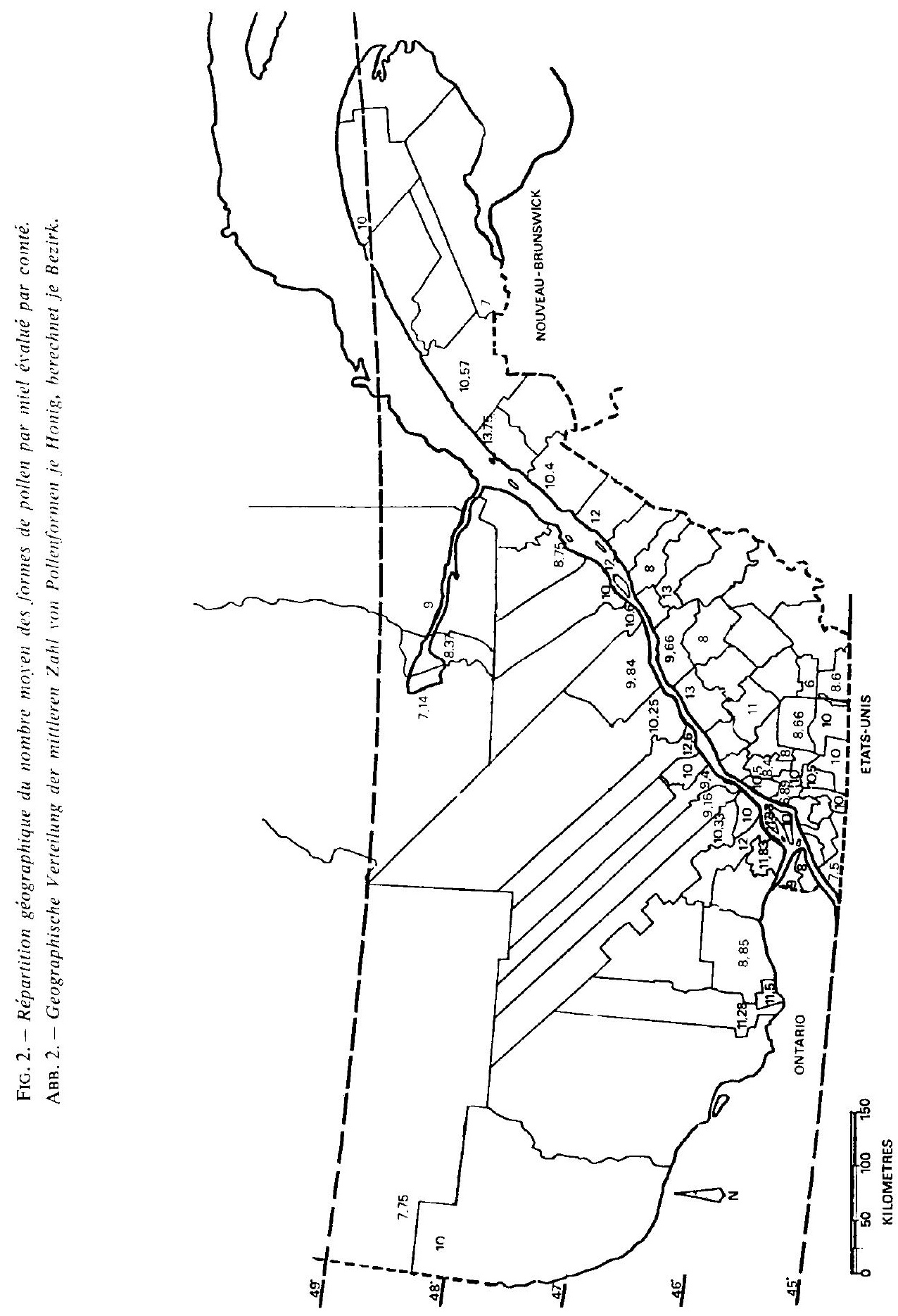


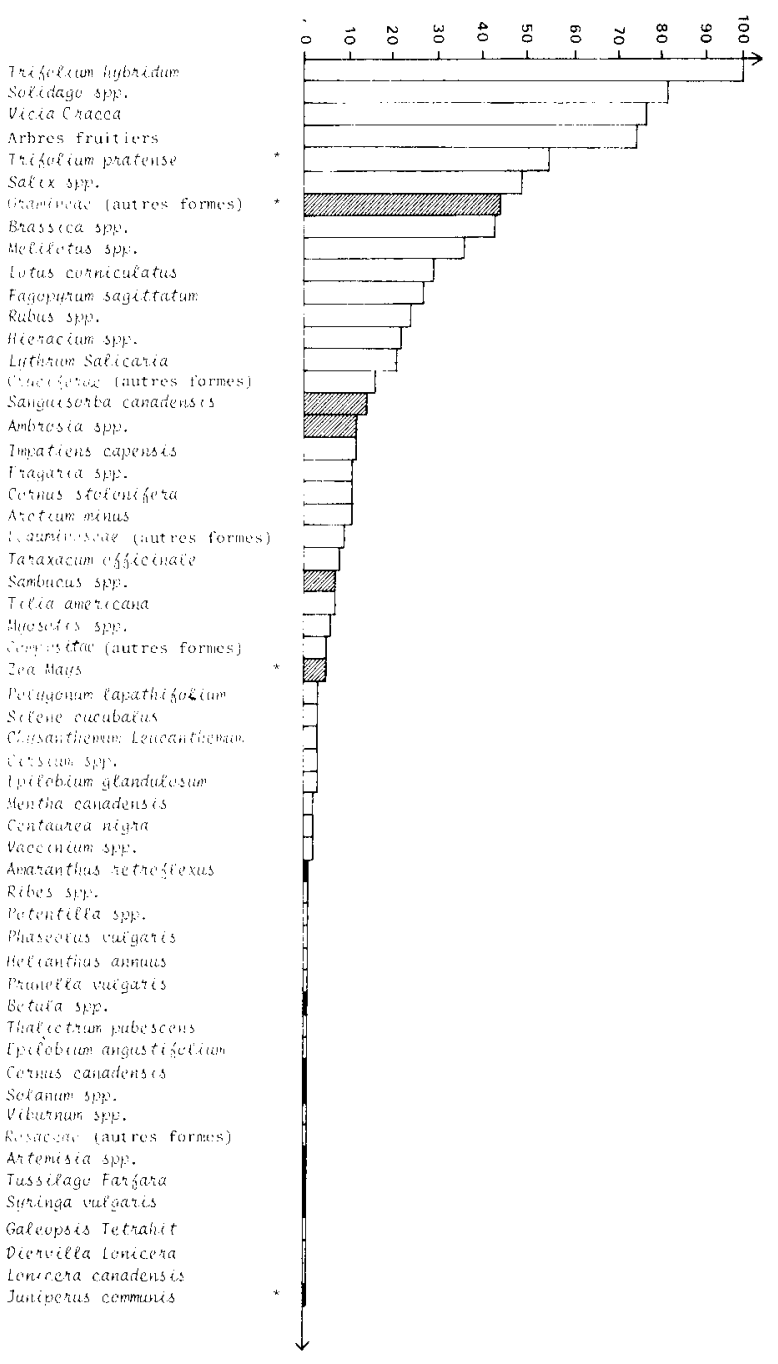

FIG. 3. - Spectre de fréquence des pollens dans les miels étudiés (en \% des échantillons étudiés).

: plantes nectarifères

: plantes seulement pollinifères

: pas de renseignements sur l'aptitude mellifère ou

plante non mellifere

* : traduit probablement une récolte de miellat

Aвв. 3. - Häufigkeitsspektrum der Pollen in den untersuchten Honigen : Nektarpflanzen

(in \% der untersuchten Proben).

\section{: Pollenpflanzen}

: Keine Angaben über die Nektarergiebigkeit oder keine

Nektarpflanzen

* : zeigt vermutlich eine Honigtauernte an. 

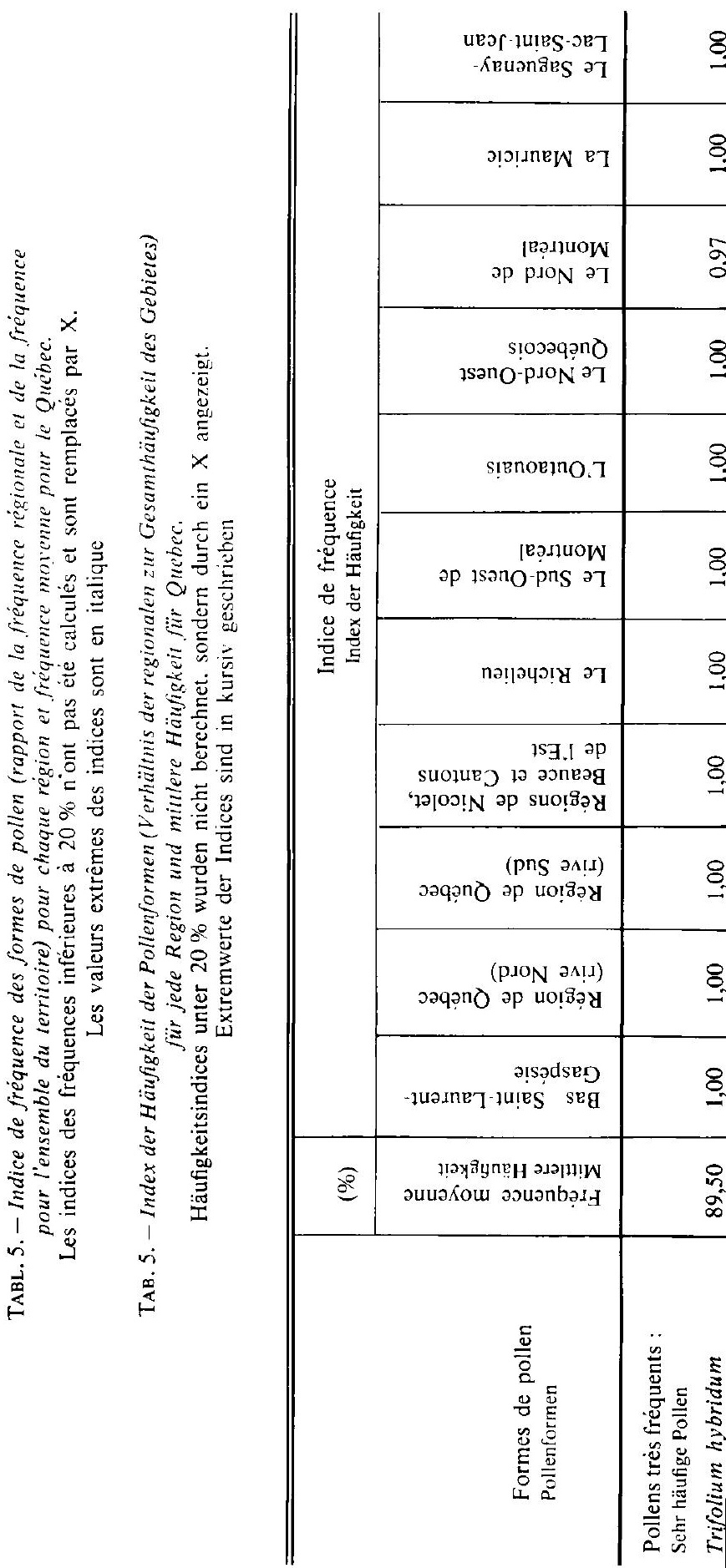


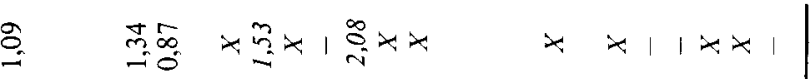

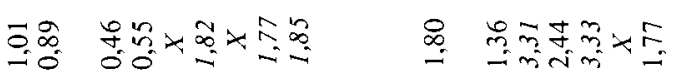

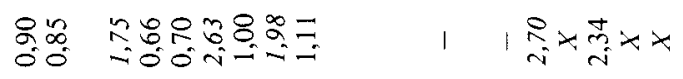

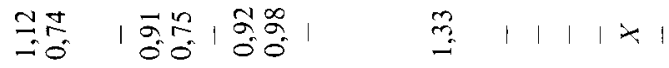

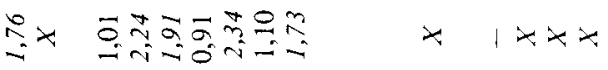

공

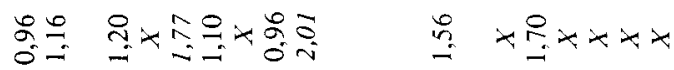

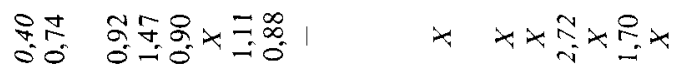

E̊

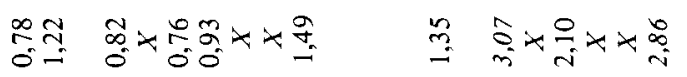

芯

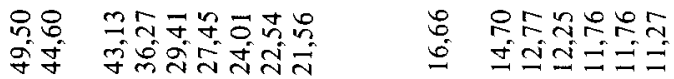

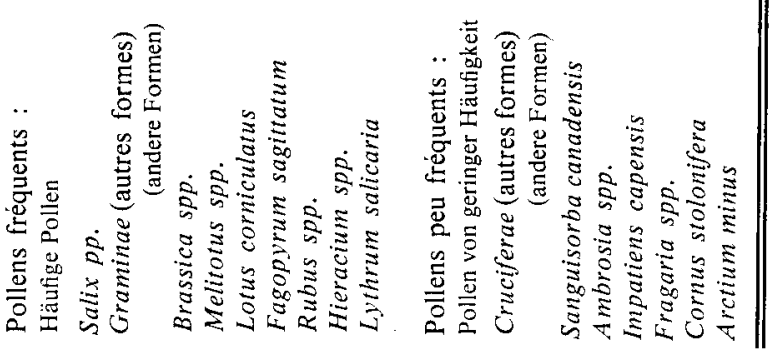


pollen pour chaque région (l'indice de fréquence est calculé en faisant le rapport de la fréquence locale par rapport à la fréquence moyenne du Québec). Si les formes du premier groupe, les pollens très fréquents, ont une dispersion à peu près égale dans les miels de toutes les régions, la disparité de répartition s'accentue avec la réduction de fréquence des formes de pollens. Déjà certaines formes de pollen du premier groupe, moins fréquentes, comme celles des arbres fruitiers et de Trifolium pratense montrent dans les miels de certaines régions quelques variations par rapport à la moyenne. Celles-ci se situent surtout dans la région de Nicolet, Beauce et des Cantons de l'Est où les formes de pollens d'arbres fruitiers (100\%), de Solidago spp. $(93,33 \%)$ et de Trifolium pratense $(86,66 \%)$ atteignent une fréquence bien au-dessus de la moyenne. Dans l'Outaouais Trifolium pratense présente une fréquence sous la moyenne $(31,25 \%)$. Les écarts les plus importants à la moyenne sont en italique dans le tableau 5. Dans le deuxième groupe, les pollens fréquents, la variation de répartition des pollens dans les miels des régions s'accentue et l'absence de certaines formes de pollen peut même être constatée par places. Il s'agit de Brassica spp. dans le Nord-Ouest québecois, de Fagopyrum sagittatum dans le Nord-Ouest québecois et au Saguenay-Lac-Saint-Jean et de Lythrum salicaria dans la région de Nicolet, Beauce et des Cantons de l'Est et dans le Nord-Ouest québecois. Dans le même groupe doit également être soulignée la fréquence particulière de certaines formes de pollen égale environ au double de la moyenne (40 à $87,5 \%$ ). Les fréquences locales inférieures à $20 \%$ n'ont pas été considérées et remplacées par un $\mathrm{X}$ dans le tableau. Dans le troisième groupe, les pollens peu fréquents, la disparité de fréquence et le nombre d'absences de certaines formes sont plus importants. La fréquence de certaines formes de pollen atteint des taux environ triples de la moyenne dans certaines régions (40 à $50 \%$ ). Pour les pollens rares, la répartition des formes dans les miels des diverses régions est très inégale. Quelques taux de fréquence locale relativement élevés doivent être soulignés. Il s'agit des formes de pollen de Légumineuses $(55,55 \%)$ et de Composées $(49,44 \%)$ dans le Nord-Ouest québecois et de Ribes spp. en Mauricie (30\%).

\section{L'abondance des formes de pollen dans les miels}

Dans le tableau 6, les formes de pollen les plus abondantes sont classees par ordre de fréquence. Les quatre catégories de fréquence y sont représentées : toutes les formes de pollen très fréquentes, environ $50 \%$ des pollens fréquents et peu fréquents et même trois formes rares. Des formes de pollen dominantes existent dans les trois premières catégories de fréquence et si elles atteignent un plus grand nombre dans la première catégorie de fréquence, elles ne se présentent pas pour toutes les formes de pollen.

\section{DISCUSSION ET CONCLUSION}

Les miels du Québec sont caractérisés principalement par quatre formes de pollen trouvées dans les miels de chaque région à des fréquences égales au moins à environ 


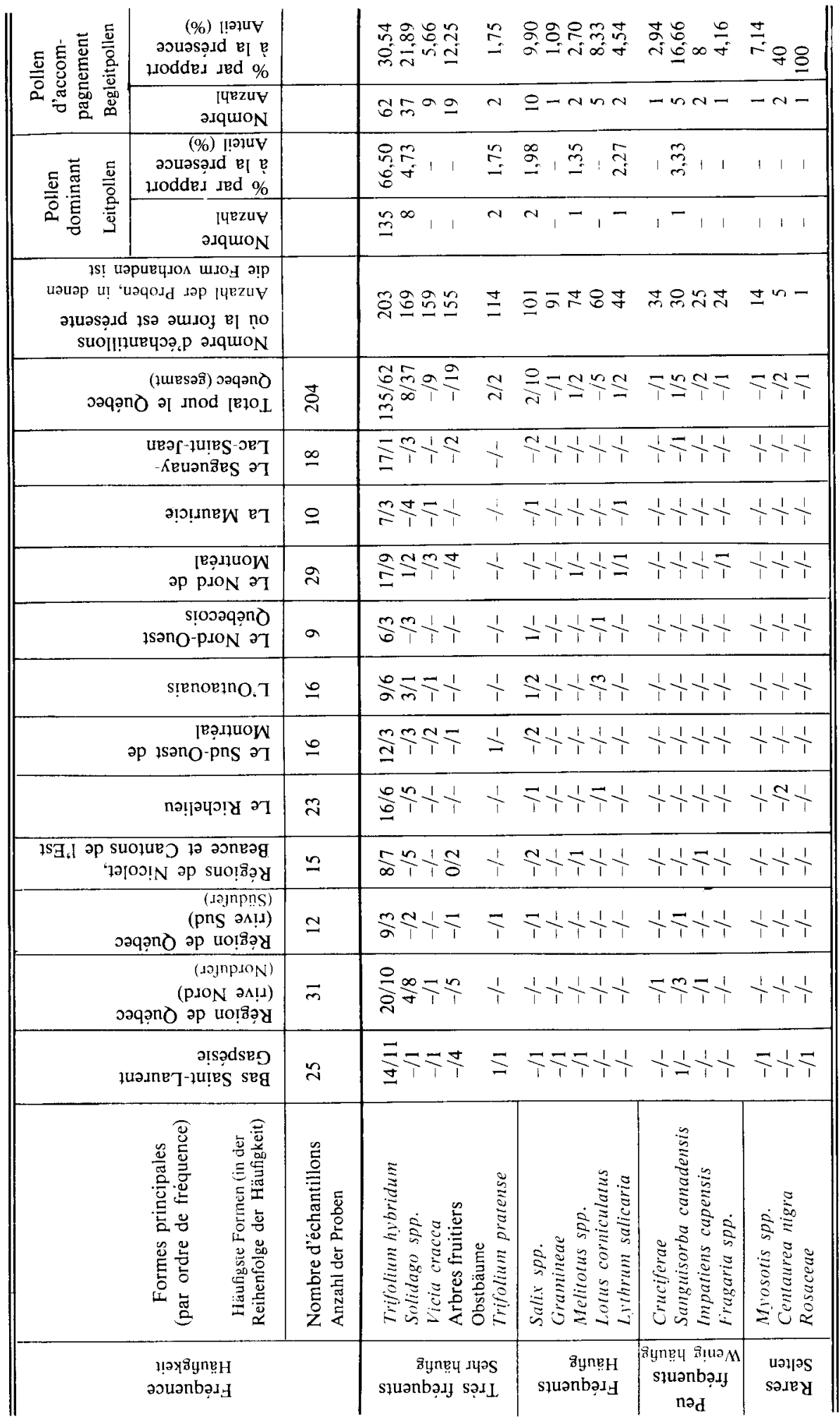


$50 \%$, il s'agit de Trifolium hybridum, Solidago spp., Vicia cracca et les arbres fruitiers et à un degré moindre par Trifolium pratense et Salix spp. dont la fréquence locale peut dans certains cas baisser jusqu'à $20 \%$. Trifolium hybridum donne lieu à la dominance la plus étendue $(66,50 \%)$, la seule répartie dans toutes les régions de la province. $\mathrm{Si}$ cette forme n'apparait pas comme pollen dominant, elle se présente comme pollen d'accompagnement $(30 \%)$ mais rarement comme pollen isolé. C'est la forme de pollen la mieux représentée dans les miels du Québec. LouveAux (1966) la considère comme la base des spectres polliniques des miels canadiens. Pellet estime que le trèfle est la meilleure plante mellifère pour l'est du Canada (1977). Par ailleurs, les miels de trèfle sont fréquents dans de nombreuses régions du monde, telles que l'Europe du Nord, la Nouvelle-Zélande, l'Est des États-Unis (MaUrizio et Louveaux, 1965). Solidago spp. se retrouve dans toutes les régions du Québec mais parait plus abondant le long de la rive Nord du Saint-Laurent et de la rivière Ottawa où se retrouvent d'ailleurs des cas de dominance. Cette constatation confirme les données de MaURizio et Louveaux (1965) qui rapportent que les miels de Solidago spp. fréquents en Amérique du Nord ne sont récoltés qu'en bordure des grands fleuves. En Europe ces miels sont rares bien que signalés dans les plaines du Danube et du Rhin (MAurizio et Louveaux, 1965). LouVEAUX (1966) signale sa présence dans la majorité des miels canadiens mais ne la considère caractéristique que pour les miels de Nouvelle Écosse. Vicia cracca est habituellement à l'état de pollen isolé tel que le signalent MaUrizio et Louveaux (1965) dans les miels d'Europe mais cette forme de pollen atteint ici quelquefois l'abondance de pollen d'accompagnement. Cette forme est pratiquement absente des miels canadiens analysés par Louveaux (1966). Les formes d'arbres fruitiers sont parfois localement assez abondantes (Bas Saint-Laurent, région de Québec rive Nord et du Nord de Montréal). Dans les comtés de Rivière du Loup et de Berthier, elles frisent la dominance. Des miels purs d'arbres fruitiers n'ont cependant pas été trouvés comme MaURIzıo et Louveaux (1965) le signalent pour l'Amérique du Nord. Bien que les miels purs de Trifolium pratense soient rares (Maurizio et Louveaux, 1965 et Louveaux, 1966), deux cas de dominance ont été détectés dans la province et deux cas de pollens d'accompagnement (Bas Saint-Laurent-Gaspésie, comté de Rimouski; Québec, rive Sud, comté de Bellechasse; Sud-Ouest de Montréal, comté de Vaudreuil). Les formes de Salix spp., dont la fréquence moyenne est légèrement inférieure à $50 \%$ présentent une abondance caractéristique dans les miels de l'ouest (Outaouais, Nord-Ouest québecois) où des cas de dominance sont retrouvés mais les cas de pollen d'accompagnement ne sont pas rares dans la province. Une telle abondance de cette forme de pollen n'a que rarement été signalée et seulement dans des régions assez humides et pour des miels de printemps (MaURizio et LouveauX, 1965). Cette observation est en contradiction avec les données de Pellet (1977) selon qui ce type de miel ne peut être récolté dans les états du Nord des États-Unis où la floraison de Salix spp. est trop précoce pour que les abeilles puissent en profiter pleinement.

Plusieurs autres formes sont indicatrices de l'origine géographique des miels. Parmi celles qui se rencontrent localement comme pollen d'accompagnement, la répar- 
tition do deux formes dans les miels correspond de façon précise à la distribution géographique de la plante. Il s'agit de Lythrum salicaria qui est abondante le long des rives du Saint-Laurent (frère Marie-Victorin, 1964; C. Rousseau, 1974) et de Sanguisorba canadensis qui est limitée à la partie orientale du Québec est rare ou inconnue ailleurs (frère MARIE-VICTORIN, 1964; C. RousseaU, 1974). Or les formes de Lythrum salicaria fréquentes dans les miels de la région de Québec rive Nord (voir tableau 5) ont une fréquence accrue dans les régions plus chaudes le long de la rive Nord du Saint-Laurent ou dans des régions plus méridionales tel le Richelieu. L'abondance de cette forme de pollen est limitée au Nord de Montréal et à la Mauricie (voir tableau 6). Sanguisorba canadensis n'a été décelée que dans les miels de la partie orientale de la province (voir tableau 5) mais avec une fréquence accrue dans le Bas SaintLaurent-Gaspésie et dans la région de Québec rive Nord. Deux formes de pollen ont une dispersion limitée dans les miels alors que la distribution géographique de la plante ne l'est pas. Ainsi, Fragaria spp. qui est un genre commun au Québec n'est cependant fréquent (voir tableau 5) et abondant que très localement ( 1 cas de pollen d'accompagnement au Nord de Montréal). Centaurea nigra, pollen rare, n'est retrouvé que dans les miels des régions situées au Sud du Saint-Laurent (voir tableau 5) et avec une certaine abondance dans le comté de Missisquoi (Richelieu), bien que la plante soit répandue sporadiquement dans toute la province mais surtout à l'est de la ville de Québec (C. Rousseau, 1968). Deux autres formes, Impatiens capensis et Myosotis spp. paraissent plutôt propres à des milieux écologiques donnés qu'à des régions géographiques particulières. Cependant les formes de Impatiens capensis, plante de milieux humides, paraissent limitées aux miels du centre et du centre-est de la province (région de Québec rive Nord, région de Québec rive Sud, Nicolet, Beauce, Cantons de l'Est et Mauricie) et les pollens de Myosotis spp. ne sont relativement fréquents que dans les miels du Bas Saint-Laurent et montrent une certaine abondance dans le comté de Kamouraska. Parmi les formes qui ne se rencontrent que comme pollen isolé, deux sont à retenir. Ambrosia spp., dont les formes sont fréquentes dans les miels du Richelieu, du Nord de Montréal et de Mauricie, est une plante commune au Québec surtout dans les régions urbaines (C. Rousseau, 1974). Cornus stolonifera dont les formes sont fréquentes dans le Bas Saint-Laurent-Gapésie, la région de Québec rive Sud et la région de Nicolet et de la Beauce est une plante transcontinentale au Canada (C. Rousseau, 1974) mais probablement plus abondante dans l'est du Québec (plusieurs cas de pollens isolés importants dans le Bas Saint-Laurent) où les tourbières, pâturages et bois de confières sont abondants.

D'autres formes sont très fréquentes localement bien que retrouvées dans les miels de presque toutes les régions de la province : les Graminées dans le Bas Saint-LaurentGaspésie, Brassica spp. dans le Bas Saint-Laurent, le Richelieu et le Nord de Montréal, Melilotus spp. dans la région de Nicolet, de la Beauce et des Cantons de l'Est, le SudOuest de Montréal, l'Outaouais et le Saguenay-Lac-Saint-Jean, Lotus corniculatus en Outaouais, dans le Sud-Est de Montréal et le Richelieu, Fagopyrum sagittatum au SudOuest de Montréal, au Nord de Montréal et en Mauricie, Rubus spp. en Outaouais et 
au Lac-Saint-Jean et Hieracium spp. au Nord de Montréal. Toutes ces formes ne se présentent que comme pollens isolés dans les miels sauf les Graminées, Melilotus spp. et Lotus corniculatus qui peuvent parfois montrer une certaine abondance localement (voir tableau 6). Toutes sont de bonnes sources de nectar sauf les Graminées, mais quelquefois dans des conditions climatiques différentes comme pour Fagopyrum sagittatum (régions humides) et Melilotus spp. (régions sèches) (PELlet, 1977). Il est à noter que la culture de Lotus corniculatus dans l'ouest de la province peut justifier la fréquence et l'abondance de cette forme de pollen dans les miels de cette région.

Quatre genres paraissent d'importance majeure pour l'apiculture dans nos échantillons : Trifolium spp., Solidago spp., les arbres fruitiers et Salix spp. Plusieurs autres sont d'importance secondaire : Vicia spp. pour l'ensemble de la province et tous les genres ou espèces dont les formes d'intérêt local ont été citées plus haut. La plupart de ces genres figurent dans les listes de plantes mellifères de СНАвот (1948) sauf Lotus corniculatus, Cornus stolonifera et Impatiens capensis. Les formes de Brassica spp. et de Fagopyrum sagittatum qu'il désigne d'importance majeure n'ont pas été trouvées en quantite très appréciable dans les miels étudiès. Les formes de Tilia americana, d'Epilobium angustifolium et de Vaccinium spp. que SLADEN (PELleT, 1977) considère entre autres comme des sources importantes de nectar au Québec, n'ont été que très rarement retrouvées. Les formes d'Acer spp. que le même auteur cite pour les récoltes de printemps sont totalement absentes des miels. Les formes trouvées d'importance majeure au Québec sont incluses parmi celles que Pellet (1977) considère comme telles pour l'ensemble de l'est du Canada depuis l'Ontario et pour l'état du Maine aux États-Unis. Le Québec ne peut dans ce cas se caractériser des régions voisines que par des formes d'importance locale telles que Lythrum salicaria et Sanguisorba canadensis.

Les familles les mieux représentées dans les miels du Québec sont les Légumineuses, tant quantitativement que qualitativement puis les Composées et enfin les Rosacées. Les Crucifères ainsi que les autres familles n'apportent qu'une part réduite dans ces miels.

La flore qui entre dans la composition des miels du Québec est principalement spontanée. Peu de formes de plantes cultivées ont été retrouvées et, à part le trèfle, souvent en nombre inférieur à ce qui peut être attendu. C'est le cas notamment des pollens d'arbres fruitiers qui n'atteignent que rarement le taux de pollen d'accompagnement et de ceux de Fagopyrum sagittatum qui ne dépassent jamais le taux de pollen isolé important. Certaines plantes spontanées, comme Vaccinium spp. n'ont pas atteint non plus l'abondance de formes attendue. Il est à noter que cette étude basée sur un échantillonnage ne peut livrer toutes les caractéristiques polliniques étant donné l'étendue du territoire couvert par l'étude et le manque de réglementation dans la récolte du miel par les apiculteurs.

Cette analyse confirme bien que la majorité des miels étudiés sont des miels mélangés. Ils contiennent des formes de pollen appartenant à la fois à des plantes à floraison printanière, estivale et automnale résultant du fait que habituellement une seule 
récolte s'effectue à l'automne et quelquefois, mais rarement, une autre dans le courant de l'été.

L'évaluation et l'interprétation de ces analyses sont délicates par suite de la carence de données phytogéographiques et d'études quantitatives de la végétation dans la province. Cette analyse permet cependant d'observer qu'il existe des caractéristiques de la composition pollinique des miels propres à chaque région. Leur examen permet de délimiter une zone médiane qui comprend les régions riveraines du Saint-Laurent et de la rivière Ottawa et qui produit des miels qui se distinguent non seulement par un plus grand nombre et une plus grande variété de formes, mais aussi par des formes typiques. Certaines d'entre elles varient d'est en ouest en fréquence et en abondance probablement par suite d'une différence de distribution géographique de la plante, de l'effet des conditions de milieu sur la production de nectar, ou de la dúrée de la période de végétation (voir figure 4). Les miels provenant des régions situées au nord et au sud de cette zone paraissent posséder les uns et les autres un nombre réduit de formes, mais diffèrent entre eux par des formes particulières. Cette étude permet ainsi l'esquisse de six grandes zones (tableau 7) dont les limites ne sont pas nécessairement précises, car les caractères se superposent à leurs frontières, ni absolues, en particulier dans les régions de Nicolet et de Beauce dont le nombre d'échantillons est réduit. Les caractéristiques générales données pour chaque zone ne se rapportent pas nécessairement à la totalité des régions qu'elles comportent. Par ailleurs, certaines particularités propres à des régions isolées n'y figurent pas. La Gaspésie a été incluse avec le Bas Saint-Laurent, mais le nombre réduit d'échantillons n'en permet pas d'évaluer les miels de façon précise. Ils ne s'écartent pas de ceux du Bas Saint-Laurent mais toutes les caractéristiques de ces derniers n'y ont pas été retrouvées. Une ébauche de cartographie de ces zones se présente dans la figure 4 où un parallèle est établi avec la durée moyenne de croissance de la végétation. Il apparait que les ruchers ne sont installés qu'en des endroits où la durée moyenne de végétation dépasse 160 jours par an sauf dans le Nord-Ouest Québécois où quelques-uns sont situés en deçà de cette limite. Cette constatation permet d'expliquer non seulement le nombre réduit de formes dans les miels de cette région, mais un spectre pollinique différent et apparemment plus pauvre par rapport à des miels provenant d'autres régions situées à la même latitude.

\section{REMERCIEMENTS}

Les auteurs remercient la Direction Générale de I'Inspection des Aliments et la Division d'Apiculture et d'Acériculture du Ministère de l'Agriculture du Québec pour leur participation à l'échantillonnage et l'encouragement apporté à cette étude. Ce travail a pu être réalisé grâce aux subventions du Conseil de Recherches et Services Agricoles du Québec et du Fonds Institutionnel de Recherches de l' l'Université du Québec à Rimouski. 

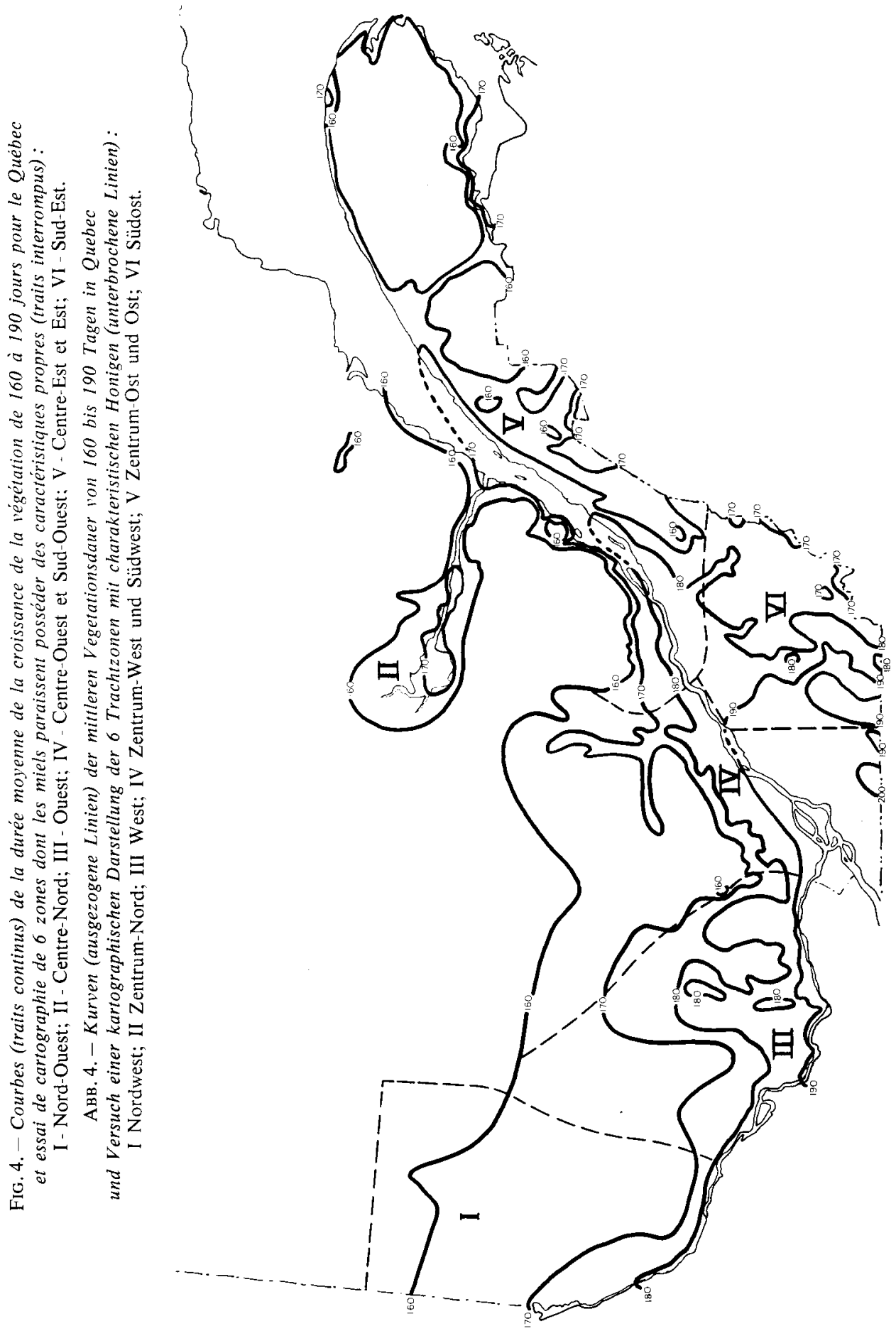


\section{ZUSAMMENFASSUNG}

Diese Untersuchung umfasst 206 Honigproben, die in den Jahren 1974 und 1975 in der gesamten Region von Quebec - d.h. überall dort, wo Bienenzucht betrieben wird. also südlich des 49 . Breitengrades - geerntet wurden (Tab. 1, Abb. 1).

Die Honige von Quebec sind im allgemeinen pollenarm. d.h. die festgestellten Pollenzahlen liegen unter den Zahlen, die man im mittleren Bereich findet ( $T a b .2$ ). Es wurden 56 verschiedene Formen gefunden. Das Pollenspektrum ist in Tab. 3 wiedergegeben. Die Kategorie mit 6-10 verschiedenen Formen dominiert (Tab. 4). Die mittlere Zahl der Pollenformen. die in den Honigen pro Bezirk gefunden wurden, ist gewöhnlich entlang des St-Lorenz-Stromes höher.

Das Studium des Häufigkeitsspektrums (Abb. 5, Tab. 5) und die quantitative Untersuchung der Pollenformen (Tab. 6) ergibt mehrere Besonderheiten.

Vier Pollenformen sind für die Bienenzucht in Quebec von besonderer Bedeutung: Trifolium spp. (Klee), Solidago spp. (Goldrute), Obstbäume und Salix spp. (Weiden).

Trifolium hybridum (Bastardklee) hat den grössten Anteil an den Honigen von Quebec. Diese sind charakterisiert durch das Vorhandensein von Solidago spp. und lokal durch verschiedene geographische Indikatoren: Lythrum salicaria (Blutweiderich), vorwiegend am Nordufer des St-Lorenz-Stroms; Sanguisorba canadensis (kanadischer Wiesenknopf) und Cornus stolonifera (Roter Hartriegel) in Ostquebec: Lotus corniculatus (Hornklee) im Westen der Provinz: Fagop.rum sagittatum (Buchweizen) in der Region von Montreal und in der Mauricie; Melilotus spp. (Riesenhonigklee) in den trockensten Gebieten abseits vom St-Lorenzstrom. Andere Formen haben eine lokale Bedeutung, sie sind jedoch weniger charakteristisch für eine bestimmte geographische Region : Myosotis spp. (Vergissmeinnicht) in Unter-St-Lorenz: Fragaria spp. (Erdbeeren) nördlich von Montreal: Impatiens capensis (Rühr-mich-nichtan vom Kap) im mittleren und mittelöstlichen Teil des Gebietes; Brassica spp. (Kohl und Senf) in Unter-StLorenz, Richelieu und nördlich von Montreal; Rubus spp. (Himbeeren) in Outaouais und Saguenay Lac St-Jean; Ambrosia spp. in Richelieu, nördlich von Montreal und Mauricie: Centaurea nigra (Schwarze Flockenblume) in Richelieu und den Nicolet-Beauce-Kantonen des Ostens, Hieracium spp. (Habichtskraut) im Norden von Montreal.

Im Lichte dieser Untersuchung lässt sich Quebec in sechs grosse Zonen einteilen, deren Honige charakteristische Merkmale zu besitzen scheinen (Tab. 7, Abb. 4).

\section{BIBLIOGRAPHIE}

Bailey L. H., 1969. - Manual of Cultivated Plants. Mc Millan.

Bureau de la Statistique du QuÉBec, 1974. - Miels 1973, Ministère de l'Industric et du Commerce. Bureau de la statistiQue du QuéBeC, 1976. - Atlas du Code Géographique du Québec. Ministère de l'Industrie et du Commerce.

Снавот, 1948. - Plantes mellifères du Québec, Ministère de l'Agriculture. Service de l'horticulture.

Document interne du Ministère de l'Agriculture du Québec, non publié

COMMISSION DE GÉOGRAPHIE, 1969. - Répertoire géographique du Québec. Ministère des Terres et Forêts.

Fernald M. L., 1950. - Gray's Manual of Botany. American Book Company.

Grantner M. M., 1966. - La végétation forestière du Québec méridional. Les Presses de l'Universite Laval.

Kerkvliet J. D. und Van der Putten A. P. J.. 1975. - Das Pollenbild einiger niederländischer Honige. Apidologie, 6, 195-206. 
Lieux M. H., 1972. - A melissopalynological study of 54 Louisiana (USA) honeys. Rev. Palaeobot. Palynol, 13, 94-124.

LiEuX M. H., 1972. - Dominant Pollen Types Recovered from Commercial Louisiana Honeys. Economic Botany, 29, 87-96.

Louveaux J., 1966. - Pollenanalyse einiger kanadischer Honige. Zeitschrift für Bienenforschung, 8 , 195-202.

Louveaux J., Maurizio A., Vorwohl G., 1970. - Methods of Melissopalynology. Bee World, 51, 125 128.

MARIE-VICTORIN, Frère, 1964. - Flore laurentienne. Presses de l'Université de Montréal.

MaUrizio A., 1951. - Pollen analysis of Honey. Bee World, 32, 1-5.

Maurizio A., 1971. - Le spectre pollinique des miels luxembourgeois. Apidologie, 2, 221-238.

Maurizio A. et Louveaux J., 1965. - Pollens de plantes mellifères d'Europe. Union des Groupements Apicoles Français. Paris.

Pellet F. C., 1977. - American Honey Plants. Dadand and Sons Publication.

Rousseau C., 1968. - Histoire, habitat et distribution de 220 plantes introduites au Québec. Naturaliste Can., 95, 49-171.

Rousseau C., 1974. - Géographie floristique du Québec/Labrador. Distribution des principales espèces vasculaires. Les Presses de l'Université Laval.

Scoggan H. J., 1978. - The flora of Canada. National Museums of Canada.

VERGERON P., 1964. - Interprétation statistique des résultats en matière d'analyse pollinique des miels. Ann. Abeille, 7, 349-364.

Vorwohl C., 1971. - Importance et but de l'analyse pollinique du miel. Apiacta, 2, 51-54.

Wilson C. V., 1971. - Le Climat du Québec, $1^{\text {re }}$ partie, Atlas climatique. Service Météorologique du Canada. 